\title{
Application of Stochastic FDTD to Holland's thin-wire method
}

\author{
Salvador G. Garcia, Senior Member, IEEE, Miguel R. Cabello, Luis D. Angulo, A. Rubio Bretones \\ Myriam G. Atienza, Student Member, IEEE, and E. Pascual-Gil, Member, IEEE
}

\begin{abstract}
This paper presents an extension of the stochastic FDTD method to predict the standard deviation of currents and charges in the Holland FDTD sub-cell method for thin wires. A discussion is presented on the effect of cross-correlation between the variables affected by uncertainty, and the variability of the observed quantities. For this, a simple dipole antenna loaded with a Wu-King profile is analyzed, and the results are validated with the Monte Carlo method.
\end{abstract}

Index Terms-FDTD, stochastic methods, Monte Carlo method, thin-wire model, Wu-King antenna.

\section{INTRODUCTION}

The Finite Difference Time Domain (FDTD) method [1] is probably the one most widely used in computational electromagnetics. It is based on the replacement of the space and time derivatives in Maxwell curl equations by secondorder accurate finite differences (and averages) [2]. With this method, we can simulate the broadband response of highly complex systems, including arbitrary materials, by an explicit marching-on-in-time algorithm which is easily parallelized in modern architecture.

The Holland's method [3] is a sub-cell technique which enables us to deal in FDTD with thin wires of radii smaller than the mesh size (less than half the space step). The current and charge (or voltage) along the wire fulfill transmission-line equations that can be solved by a 1D FDTD method. They are coupled with the Yee 3D FDTD in a full-wave two-way procedure, exchanging information in the form of independent sources. Holland's method has been extended to deal with multiwires including lumped circuits at wire-wire and wirestructure junctions [4], complex cable bundles [5], oblique wires [6] [7], carbon nanotube antennas [8], etc..

Several approaches exist to cope with the prediction of the uncertainty in FDTD calculations [9]. Among these, the stochastic FDTD method (S-FDTD) presented in [10] employs a FDTD-like algorithm, in order to find also the standard deviation $\sigma$ on the fields, as a function of the $\sigma$ of the material constitutive parameters. S-FDTD is a single-realization algorithm, as opposed to the classical Monte Carlo (MC) FDTD method, which uses a brute-force approach to find the

The work described in this paper and the research leading to these results have been supported by the Spanish MINECO, EU FEDER under Project TEC2016-79214-C3-3-R (MINECO, Spain), and the Alhambra-LFT contract with AIRBUS (Spain).

The five first authors are with the Dept. of Electromagnetism, University of Granada, Fuentenueva s/n, 18071 Granada, Spain. The last one is with Airbus, P. John Lennon s/n, 28906 Getafe, Spain. Corresponding author email: salva@ugr.es statistical parameters from the simulation of a set of problems (often several thousand) with material properties randomly generated. The stochastic methodology has been extended to deal with transmission lines [11], to the finite difference frequency domain method [12], etc.

In this paper, we present an extension of the S-FDTD method to the Holland's thin-wire subcell model, and we describe a simple implementation in existing MPI FDTD codes. The proposed method is validated by modeling a $\mathrm{Wu}-$ King loaded antenna [13] with statistical variations on its resistive load values.

The rest of this paper is organized as follows. Section II briefly reviews the fundamentals of Holland's thin-wire model. Section III describes the extension of S-FDTD to the coupled algorithm Holland FDTD/Yee FDTD. Section IV provides a quick description of an easy implementation of S-FDTD in existing FDTD MPI codes. Section V applies this new approach to a simple Wu-King loaded dipole, and we conclude in Section VI with some remarks on the applicability of the S-FDTD method depending on the correlation coefficient between the load values at different space positions. Specifically, we show that the results agree with MC-FDTD only when perfect correlation exits between the loads at different space points. We also show that $\sigma$, for the random noncorrelated case, can be determined by performing one S-FDTD simulation per each load with non-null $\sigma$, and combining the results.

\section{HOLLAND'S SUBCELL METHOD FOR THIN WIRES}

The Yee FDTD scheme solves Maxwell curl equations by placing the electric and magnetic field components staggered in space and time by half space and time steps, and replacing the derivatives by finite differences. It finds the unknown field components at a given time step as a function of them at earlier times and at adjacent space positions. For instance, for the $E_{z}$ component ${ }^{1}$

$$
\begin{aligned}
& E_{z_{i, j, k}}^{n+1 / 2}=C_{a_{i, j, k}} E_{z_{i, j, k}}^{n-1 / 2}+C_{b_{i, j, k}}\left(J_{z_{i, j, k}}^{n}+\right. \\
& \left.\frac{H_{y_{i+1 / 2, j, k}}^{n}-H_{y_{i-1 / 2, j, k}}^{n}}{\Delta x}-\frac{H_{x_{i, j+1 / 2, k}}^{n}-H_{x_{i, j-1 / 2, k}}^{n}}{\Delta y}\right)
\end{aligned}
$$

where $C_{a}$ and $C_{b}$ are constants depending on the permittivity $\varepsilon$ and the conductivity $\sigma$

$$
C_{a_{i, j, k}}=\frac{2 \varepsilon_{i, j, k}-\Delta t \sigma_{i, j, k}}{2 \varepsilon_{i, j, k}+\Delta t \sigma_{i, j, k}}, C_{b_{i, j, k}}=\frac{2 \Delta t \varepsilon_{i, j, k}}{2 \varepsilon_{i, j, k}+\Delta t \sigma_{i, j, k}}
$$

${ }^{1}$ We follow a notation similar to that of [14], but shifting the usual indexes $i, j$ by half an increment, for the ease of writing of this manuscript. 
The Holland's formulation for wires thinner than the FDTD mesh step, discretizes the wire along its length, and finds the current $I$, and the charge $Q$ per unit length (p.u.1.), by transmission-line equations. For instance, for a wire aligned along the $\mathrm{z}$ direction

$$
\begin{aligned}
\frac{\partial I}{\partial t}+\frac{R}{L} I+c^{2} \frac{\partial Q}{\partial z} & =\frac{1}{L}\left\langle E_{z}\right\rangle \\
\frac{\partial Q}{\partial t}+\frac{\partial I}{\partial z} & =0
\end{aligned}
$$

where $R$ and $L$ are the p.u.l. resistance and inductance, and $c$ is the free-space light speed. $L$ consists on the sum of a given arbitrary term, and an intrinsic term (in-cell inductance), this being found by some average across the FDTD cell perpendicular to the wire direction [3] resulting in

$$
L_{\text {intr }}=f(a, \Delta x, \Delta y)
$$

where $a$ is the wire radius, and $\Delta u$ the usual FDTD space steps. Similarly, $\left\langle E_{z}\right\rangle$ is an average value of the E-field over a surface perpendicular to the wire direction, usually simply taken as the point-wise usual $E_{z}$ field component found by Maxwell equations if the wire path runs along FDTD electric edges.

With the application of a 1D FDTD scheme to Eqs. (3)(4), an explicit advancing algorithm is found at wire locations $x=$ $i_{0} \Delta x, y=j_{0} \Delta y, z=k \Delta z$

$$
\begin{aligned}
& I_{k}^{n+1}=G_{a_{k}} I_{k}^{n}+G_{b_{k}}\left(Q_{k-1 / 2}^{n+1 / 2}-Q_{k+1 / 2}^{n+1 / 2}\right)+G_{c_{k}} E_{z_{i_{0}, j_{0}, k}}^{n+1 / 2} \\
& Q_{k+1 / 2}^{n+1 / 2}=Q_{k+1 / 2}^{n-1 / 2}+\frac{\Delta t}{\Delta z}\left(I_{k}^{n}-I_{k+1}^{n}\right)
\end{aligned}
$$

with

$$
\begin{gathered}
G_{a_{k}}=\frac{2 L_{k}-R_{k} \Delta t}{2 L_{k}+R_{k} \Delta t}, \quad G_{b_{k}}=\frac{c^{2}}{\Delta z} \frac{2 L_{k} \Delta t}{2 L_{k}+R_{k} \Delta t} \\
G_{c_{k}}=\frac{2 \Delta t}{2 L_{k}+R_{k} \Delta t}
\end{gathered}
$$

Eqs. (6) are coupled to FDTD Eq. (1) in a two-way manner: the value $E_{z_{i_{0}, j_{0}, k}}^{n+1 / 2}$ is taken from (1) and plugged into (6), playing the role of distributed voltage sources, while the currents from (6) are injected back into (1), playing the role of current density sources $J_{z_{i_{0}, j_{0}, k}}^{n}=\frac{I_{k}^{n}}{\Delta x \Delta y}$.

\section{S-FDTD FOR THIN-WIRE EQUATIONS}

The impact of the uncertainty of the circuital properties on the uncertainty of the currents flowing along the thin wire, can be predicted by extending the S-FDTD method of [10] to Holland's equations. The mean values $\mu\{I\}, \mu\{Q\}$, henceforth plainly noted as $I$ and $Q$, are proven to be advanced by $\mathrm{S}$ FDTD also with Eqs. (6). Their standard deviation $(\sigma)$ is found by using the Delta method, which states in general

$$
\begin{gathered}
\sigma^{2}\left\{f\left(x_{1}, \ldots, x_{N}\right)\right\} \simeq \sum_{\forall i}\left(\left.\frac{\partial f}{\partial x_{i}}\right|_{\mu\left\{x_{1}\right\}, \ldots}\right)^{2} \sigma^{2}\left\{x_{i}\right\}+ \\
\left.\sum_{\forall i, j, i \neq j} 2\left(\frac{\partial f}{\partial x_{j}} \frac{\partial f}{\partial x_{i}}\right)\right|_{\mu\left\{x_{1}\right\}, \ldots} \sigma\left\{x_{i}\right\} \sigma\left\{x_{j}\right\} \rho_{x_{i}, x_{j}}
\end{gathered}
$$

Applying the Delta method to all the additive and multiplicative terms in Eqs. (6), we find a set of advancing equations for $\sigma\{I\}, \sigma\{Q\}$ formally identical to (6) but differing in the addition of a set of independent sources, which in turn depend on the mean values previously found by Eqs. (6)

$$
\begin{gathered}
\sigma\left\{I_{k}^{n+1}\right\}=G_{a_{k}} \sigma\left\{I_{k}^{n}\right\}+G_{b_{k}}\left(\sigma\left\{Q_{k-1 / 2}^{n+1 / 2}\right\}-\sigma\left\{Q_{k+1 / 2}^{n+1 / 2}\right\}\right)+ \\
D_{a_{k}} I_{k}^{n}+D_{b_{k}}\left(Q_{k-1 / 2}^{n+1 / 2}-Q_{k+1 / 2}^{n+1 / 2}\right)+ \\
G_{c_{k}} \sigma\left\{E_{z_{i_{0}, j_{0}, k}}^{n}\right\}+D_{c_{k}} E_{z_{i_{0}, j_{0}, k}}^{n} \\
\sigma\left\{Q_{k+1 / 2}^{n+1 / 2}\right\}=\sigma\left\{Q_{k+1 / 2}^{n-1 / 2}\right\}+\frac{\Delta t}{\Delta x}\left(\sigma\left\{I_{k}^{n}\right\}-\sigma\left\{I_{k+1}^{n}\right\}\right)
\end{gathered}
$$

The constants $G_{\nu_{k}}$ are given by Eqs. (7), while $D_{a_{k}}, D_{c_{k}}, D_{b_{k}}$ $(\nu=\{a, b, c\})$ are found by

$D_{\nu_{k}} \equiv \sigma\left\{G_{\nu_{k}}\right\}=\frac{\partial G_{\nu_{k}}}{\partial L_{k}} \sigma\left\{L_{k}\right\} \rho_{L_{k}, I_{k}}+\frac{\partial G_{\nu_{k}}}{\partial R_{k}} \sigma\left\{R_{k}\right\} \rho_{R_{k}, I_{k}}$

Note that the expression above can be easily extended to take into account the uncertainties in the wire radius, by applying the same rule to find $\sigma\left\{L_{\text {intr, } k}\right\}$ from Eq. (5).

The 3D S-FDTD updating equations for $\sigma\left\{E_{z}\right\}$ must also be modified by including the $\sigma\{I\}$ of the currents. Hence, the S-FDTD counterpart of (1) becomes

$$
\begin{aligned}
\sigma\left\{E_{z_{i, j, k}}^{n+1 / 2}\right\}= & C_{a_{i, j, k}} \sigma\left\{E_{z_{i, j, k}}^{n-1 / 2}\right\}+C_{b_{i, j, k}}\left(\frac{\sigma\left\{I_{k}^{n}\right\}}{\Delta x \Delta y}+\right. \\
& \frac{\sigma\left\{H_{y_{i+1 / 2, j, k}}^{n}\right\}-\sigma\left\{H_{y_{i-1 / 2, j, k}}^{n}\right\}}{\Delta x}- \\
& \left.\frac{\sigma\left\{H_{x_{i, j+1 / 2, k}}^{n}\right\}-\sigma\left\{H_{x_{i, j-1 / 2, k}}^{n}\right\}}{\Delta y}\right)
\end{aligned}
$$

where the term $\frac{\sigma\left\{I_{l}^{n}\right\}}{\Delta x \Delta y}$ is added only at the wire locations $\left(i_{0}, j_{0}, k\right)$. No independent-source terms depending on the mean value of the fields need to be added to Eq. (12), since in this paper no uncertainty is assumed to exist on the material properties of the surrounding space.

The choice of cross-correlation coefficients deserves some discussion, since these parameters are generally not known beforehand. The S-FDTD procedure followed to find Eqs. (9)(10) reasonably assumes a good correlation between fields at close space positions ${ }^{2}$. In our case, this translates into $\rho_{I_{k}, I_{k-1}} \simeq$ $1, \rho_{Q_{k+1 / 2}, Q_{k-1 / 2}} \simeq 1$. It also typically assumes good correlation in multiplicative terms; in our case between unknowns and wire parameters $\rho_{R_{k}, I_{k}} \simeq 1, \rho_{L_{k}, I_{k}} \simeq 1$.

It can also be demonstrated that a good correlation is also implicit between wire parameters at adjacent space locations (e.g. $\rho_{R_{k}, R_{k-1}}$ ). This has impact on the comparison with the MC method, as will be shown in Section V. The latter can be appreciated if we track down the iterative process of Eqs. (9)(10). Let us just write one of the terms,

$$
\sigma\left\{I_{k}^{n}\right\}=\ldots\left(D_{b_{k}} G_{c_{k-1}}+D_{c_{k-1}} G_{b_{k}}\right) \sigma\left\{I_{k-2}^{n-2}\right\} \ldots
$$

${ }^{2}$ Other choices can also be taken into account (see [10]). 


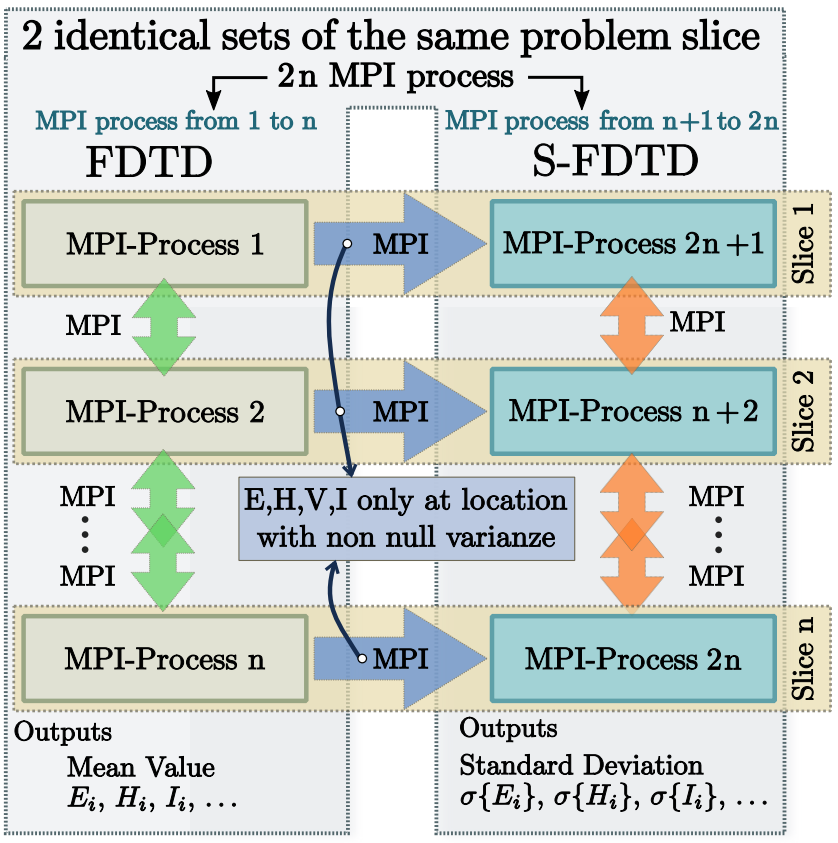

Fig. 1. Workflow of the implementation of S-FDTD using a MPI paradigm. The green, orange, and blue arrows show the MPI-comunications between the MPI process.

and its counterpart from Eqs. (6),

$$
I_{k}^{n}=\ldots\left(G_{b_{k}} G_{c_{k-1}}\right) I_{k-2}^{n-2} \ldots
$$

Taking the standard deviation of (14), and following the same procedure used to yield (11), we get (13), only if we assume unit correlation between the material parameters at adjacent cells $\rho_{G_{b_{k}}, G_{c_{k-1}}}=1$. This observation agrees with the discussion of [15] and the work of [9].

\section{MPI IMPLEMENTATION}

The computer implementation of the S-FDTD algorithm into a FDTD program already coded with a MPI paradigm is straightforward in general. For this, we take advantage of the full similarity between S-FDTD and the usual FDTD schemes, and the fact that S-FDTD only uses FDTD values as independent sources at each time step. First, two groups of MPI communicators are created: one for the FDTD domain, and another for the S-FDTD domain, which run simultaneously. Each group, in turn, divides the geometry into slices along the z-direction which communicate in the usual manner (as described in [16]). At the end of each iteration, the FDTD slice sends the magnitudes to the S-FDTD corresponding slice, to be added as independent sources. The whole procedure is depicted in Fig. 1.

In particular, the results shown in the next section were found with the MPI-OMP SEMBA-UGRFDTD code [17] using this methodology to incorporate S-FDTD with a minimum effort: no drastic changes in the algorithm, no code duplication, the same absorbing conditions, etc..

\section{VALIDATION With A Dipole ANTENNA}

Let us illustrate the application of the method presented above to the analysis of the uncertainty on the current $I_{0}$ at the center of a wire dipole antenna loaded with a Wu-King resistive profile [13]

$$
R(z)=\frac{R_{0}}{l / 2-|z|},-l / 2<z<l / 2
$$

The antenna, consisting of a thin wire of $0.2 \mathrm{~cm}$ radius, and length $l=41 \mathrm{~m}$, is discretized into segments of $\Delta z=1 \mathrm{~m}$, numbered from $k=-20$ to $k=20$. It is placed in a PMLtruncated free-space 3D Yee FDTD region, with a uniform and isotropic mesh with $\Delta x=\Delta y=\Delta z=1 \mathrm{~m}$. The antenna is fed at its center segment $k=0$ with a matched voltage source having a Gaussian broadband profile, and $R_{0}=50 \Omega$ of internal resistance. The resistance at each segment is taken by averaging Eq. (15) along the segment length.

For the simplicity of our analysis, only the two segments immediately above and below the feeding segment are assumed to have a non-null standard deviation taken as $\sigma\left\{R_{k}\right\}=0.2 R_{k}$ with $k \in\{-2,-1,1,2\}$.

First, we generate two different MC populations of $N=$ 25000 simulations each, with a normal distribution: one of them assuming random non-correlated values $\rho_{R_{k}, R_{k^{\prime}}}=$ $0, k \neq k^{\prime} \in\{-2,-1,1,2\}$, and another with well-correlated values $\rho_{R_{k}, R_{k^{\prime}}}=1$. The mean value of the current $\mu\left\{I_{0}\right\} \equiv$ $I_{0}$, and its $\sigma\left\{I_{0}\right\}$ is found in the usual way,

$\mu\left\{I_{0}\right\}=\frac{1}{N} \sum_{m=1}^{N} I_{0_{m}}, \sigma\left\{I_{0}\right\}=\sqrt{\frac{1}{N} \sum_{m=1}^{N}\left(I_{0_{m}}-\mu\left\{I_{0}\right\}\right)^{2}}$

where the subscript $m$ stands for the value found from the $\mathrm{m}$-th MC-FDTD simulation.

For S-FDTD, we have analyzed two different scenarios: the first one $\left(\mathrm{S}_{-} \mathrm{FDTD}_{1}\right)$ running a single simulation with non-null standard deviation simultaneously for all the four resistances $\sigma\left\{R_{k}\right\} \neq 0, \forall k \in\{-2,-1,1,2\}$ (inherently with unit correlation among them); and the second one (S-FDTD 4$)$ running four different simulations, assuming that only one of the resistances has non-null standard deviation at a time, and setting the other three standard deviations equal to zero $\sigma\left\{R_{k}\right\} \neq 0, \sigma\left\{R_{k^{\prime}}\right\}=0, \forall k^{\prime} \neq k \in\{-2,-1,1,2\}$.

Fig. 2 compares the absolute value of $\sigma\left\{I_{0}\right\}$ for MCFDTD, S-FDTD 4 , and S-FDTD ${ }_{1}$. S-FDTD 1 directly finds $\sigma$ from the simulation (green line). For S-FDTD 4 two alternative calculations are considered in the post-processing:

1) $\mathrm{S}-F D T D_{4}$ Uncorrelated (red line):

$$
\sigma\left\{I_{0}\right\}=\sqrt{\left.\sum_{k=-2,-1,1,2} \sigma^{2}\left\{I_{0}\right\}\right|_{\sigma\left\{R_{k}\right\} \neq 0, \sigma\left\{R_{k^{\prime}}\right\}=0, \forall k^{\prime} \neq k}}
$$

2) $\mathrm{S}-F D T D_{4}$ Correlated (blue line):

$$
\sigma\left\{I_{0}\right\}=\sum_{k=-2,-1,1,2} \sqrt{\left.\sigma^{2}\left\{I_{0}\right\}\right|_{\sigma\left\{R_{k}\right\} \neq 0, \sigma\left\{R_{k^{\prime}}\right\}=0, \forall k^{\prime} \neq k}}
$$

Reasoning in terms of the variance $\sigma^{2}$, we conclude that the variance found with the MC non-correlated population coincides with that of the S-FDTD 4 uncorrelated red-line results. This is reasonable because Eq. (17) actually finds 


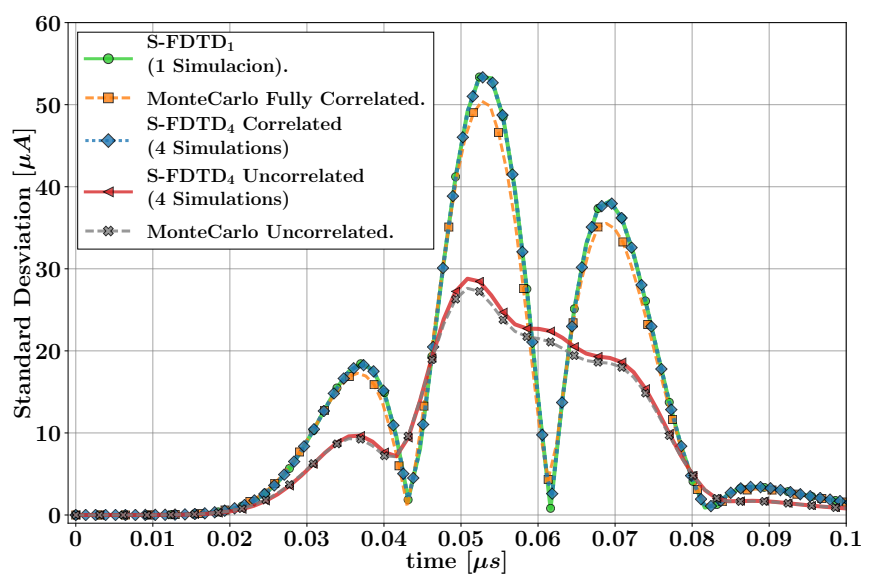

Fig. 2. Comparison of the absolute value of the standard deviation of the current at the center of a Wu-King loaded dipole, computed by the MC and S-FDTD methods.

the variance by summing each individual-simulation variance, which is the usual formula of the variance of a sum for noncorrelated data. On the other hand, the variance found with the MC fully-correlated population coincides with that of the

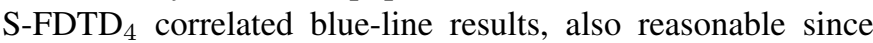
Eq. (18) actually finds the variance by the usual formula of the variance of a sum, assuming correlated data. Finally, note that the agreement of the $\mathrm{S}-F D T D_{1}$ single-simulation results with the $\mathrm{S}-F D T D_{4}$ correlated (blue-line) results, can be deduced from the linearity of the equations: the response of the system to the sum of different sources is the sum of their single responses. This corroborates that the usual S-FDTD method actually assumes unit correlation between the material parameters affected by uncertainty, as already claimed.

In any case, a more proper choice of the correlation coefficients $\rho_{R_{k}, I_{k}}$ in $\mathrm{S}_{-\mathrm{FDTD}}$, could be found by applying the method of [18], to correct Eq. (11) according to Eq.(8). An extended work, including this topic, is left for a full-length forthcoming paper.

\section{CONCLUSIONS}

Here we present a method to analyze thin-wire structures by the S-FDTD method, and study its validity depending on the assumption of cross-correlation between material parameters at different space locations. For this, we have reproduced the two extreme scenarios with the MC method: full correlation between the material parameters everywhere (four resistances in our case), and no correlation between them. A simple postprocessing after the S-FDTD simulations, isolating the variance of each parameter at a time, enables us to find the fully uncorrelated case, with a dramatic reduction in computational requirements compared with $\mathrm{MC}$, requiring just one simulation per cell or group of cells with non-null uncertainty.

On the other hand, we show that a single-simulation of S-FDTD with all parameters having a non-null variance is equivalent to performing a $\mathrm{MC}$ analysis with unit correlation between the parameters everywhere. Hence, S-FDTD results may be realistic for single-material situations, or for materials with correlated variations (e.g. due to ageing). Total random media would require a single S-FDTD simulation per random material. This could be more (or less) computationally intense than a brute-force MC approach, depending on the number of random parameters compared to the size of the population required for MC multiple realization. In most practical applications, the approach based on a single S-FDTD simulations per random parameter, may achieve dramatic reductions with respect to the MC approach.

\section{REFERENCES}

[1] K. Yee, "Numerical solution of initial boundary value problems involving Maxwell's equations in isotropic media," IEEE Transactions on Antennas and Propagation, vol. 14, no. 3, pp. 302-307, May 1966.

[2] A. Taflove and S. C. Hagness, Computational Electrodynamics The Finite-Differences Time Domain Method. Artech House, 2005.

[3] R. Holland and L. Simpson, "Finite-difference analysis of EMP coupling to thin struts and wires," Electromagnetic Compatibility, IEEE Transactions on, vol. EMC-23, no. 2, pp. 88 -97, may 1981.

[4] J. P. Berenger, "A multiwire formalism for the FDTD method," IEEE Transactions on Electromagnetic Compatibility, vol. 42, no. 3, pp. 257 264, Aug. 2000.

[5] G. G. Gutierrez, D. Mateos, M. R. Cabello, E. Pascual-Gil, L. D. Angulo, and S. G. Garcia, "On the design of aircraft electrical structure networks," IEEE Transacions on Electromagnetic Compatibility, vol. 58, no. 2, pp. 401 - 408, March 2016.

[6] C. Guiffaut, A. Reineix, and B. Pecqueux, "New oblique thin wire formalism in the FDTD method with multiwire junctions," Antennas and Propagation, IEEE Transactions on, vol. 60, no. 3, pp. 1458-1466, March 2012.

[7] J. Bérenger, "Origin of parasitic solutions with holland and simpson thin wires in the FDTD grid," IEEE Transactions on Electromagnetic Compatibility, vol. 61, no. 2, pp. 487-494, Apr. 2019.

[8] H. Lin, M. Pantoja, S. Garcia, A. Bretones, and R. Martin, "An FDTD thin-wire model for modeling carbon nanotube dipoles at thz regime," Antennas and Wireless Propagation Letters, IEEE, vol. 11, pp. 708-711, 2012.

[9] A. Ajayi, P. Ingrey, P. Sewell, and C. Christopoulos, "Direct computation of statistical variations in electromagnetic problems," IEEE Transactions on Electromagnetic Compatibility, vol. 50, no. 2, pp. 325-332, May 2008.

[10] S. M. Smith and C. Furse, "Stochastic fdtd for analysis of statistical variation in electromagnetic fields," IEEE Transactions on Antennas and Propagation, vol. 60, no. 7, pp. 3343-3350, July 2012.

[11] C. Salis, T. Zygiridis, P. Sarigiannidis, and N. Kantartzis, "Unconditionally-stable time-domain approach for uncertainty assessment in transmission lines," in 2016 5th International Conference on Modern Circuits and Systems Technologies (MOCAST), May 2016, pp. $1-4$.

[12] K. Masumnia-Bisheh, K. Forooraghi, and M. Ghaffari-Miab, "Electromagnetic uncertainty analysis using stochastic fdfd method," IEEE Transactions on Antennas and Propagation, vol. 67, no. 5, pp. 32683277, May 2019.

[13] T. Wu and R. King, "The cylindrical antenna with nonreflecting resistive loading," IEEE Transactions on Antennas and Propagation, vol. 13, no. 3, pp. 369-373, May 1965.

[14] A. Taflove, Computational Electrodynamics: The Finite-Difference TimeDomain Method. Boston: Artech House, 1995.

[15] T. Tan, A. Taflove, and V. Backman, "Single realization stochastic fdtd for weak scattering waves in biological random media," IEEE Transactions on Antennas and Propagation, vol. 61, no. 2, pp. 818828, Feb 2013.

[16] R. Mittra, Computational electromagnetics: recent advances and engineering applications. Springer Science \& Business Media, 2014.

[17] "SEMBA-UGRFDTD." [Online]. Available: http://www.ugrFDTD.com

[18] K. M. Bisheh, B. Z. Gatabi, and S. M. H. Andargoli, "Stochastic fdtd accuracy improvement through correlation coefficient estimation," Waves in Random and Complex Media, vol. 25, no. 2, pp. 154-169, 2015. [Online]. Available: https://doi.org/10.1080/17455030.2014.999845 